\title{
RELATIVISTIC EFFECTS IN CRYSTALS
}

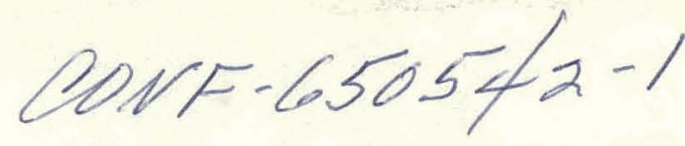

T. L. Loucks

Institute for Atomic Research and Department of Physics

Iowa State University, Ames, Iowa

Manuscript for a presentation delivered at the Midwest Theoretical Conference (1965) and to appear in the Proceedings of the Midwest Theoretical Conference (1965) Ohio State University, Columbus, Ohio.

\section{$\underline{\text { Relativistic Effects in Crystals }}$}

I would like to direct your attention to the subject of relativistic effects in crystals. In particular, I will be most interested in relativistic effects on the Fermi surfaces of metals. Theoretically, the problem can be stated quite simply: we are interested in finding Bloch functions which satisfy the Dirac hamiltonian (Slide 1) where V is the crystal potential and has the periodicity of the lattice.

In atomic physics the approach frequently taken to study relativistic effects is to use the simplified hamiltonian (Slide 2) which results from two successive applications of the Foldy-Wouthuysen transformation to the Dirac hamiltonian. One obtains three relativistic correction terms in addition to the usual kinetic and potential energy operators; spin-orbit,

\footnotetext{
Work was performed at the Ames Laboratory of the U. S. Atomic Energy Commission, Contribution No. 1732.
} 


\section{DISCLAIMER}

This report was prepared as an account of work sponsored by an agency of the United States Government. Neither the United States Government nor any agency Thereof, nor any of their employees, makes any warranty, express or implied, or assumes any legal liability or responsibility for the accuracy, completeness, or usefulness of any information, apparatus, product, or process disclosed, or represents that its use would not infringe privately owned rights. Reference herein to any specific commercial product, process, or service by trade name, trademark, manufacturer, or otherwise does not necessarily constitute or imply its endorsement, recommendation, or favoring by the United States Government or any agency thereof. The views and opinions of authors expressed herein do not necessarily state or reflect those of the United States Government or any agency thereof. 


\section{DISCLAIMER}

Portions of this document may be illegible in electronic image products. Images are produced from the best available original document. 


\section{LEGAL NOTICE}

This report was prepared as an account of Government sponsored work. Netther the United States, nor the Commission, nor any person acting on bebalf of the Commission:

A. Makes any warranty or representation, expressed or limpled, with respect to the accuracy, completeness, or usefulness of the information contained in this report, or that the use of any informats arpar

B. Assumes any liabiltiles will respect to the ubo of, or for damagea ranulting from the ef any information, apparatus, method, or process disclosed in this report.

As used in the above, "person acting on behalf of the Commission" Includes any employee or contractor of the Cor thelon, or employee of such contractor, to the extent that disseminates, or provides access to, any intormation pursausul w illa emplogment or contract with the Commission, or hts employment with such contractor. 
Darwin and mass-velocity terms. It is customary to include these relativistic terms by a perturbation calculation. A similar approach was used in some of the earlier work on relativistic effects in crystals. The problem which arises in the crystalline case is that wave functions are not readily available, even for the unperturbed state. Much of our information on the electronic states of crystals appears as energy bands or constant energy surfaces. A lot of effort has gone into finding eigenvalues, but only the very brave concern themselves with eigenfunctions (and even then only in moderation).

To mention a few examples of this perturbation approach, we might start with the work of F. Herman ${ }^{(1,2)}$ on semi-conductors (Slide 3). Here we can see the individual contributions of each of the three correction terms. These results are based primarily on atomic calculations which were adjusted in a rather arbitrary manner to account for the crystal field effects. This avoided the need for crystal wave functions, but at the same time left the method open to question. A far better approach was taken by L. Mattheiss ${ }^{(3)}$ in considering the effects of the spin-orbit interaction on the energy bands of tungsten (Slide 4). The $k$-dependence of the spin-orbit interaction in the crystal was determined in this calculation by using a tight-binding approximation for the crystal wave functions. In order to make the method practical, however, it was necessary to introduce a spin-órbit parameter which was arbitrarily adjusted in order to give agreement with experimental results. Even so, the calculation demonstrated relearly that the spin-orbit interaction could 
account for the splitting of degenerate levels which"had been observed experimentally ${ }^{(4)}$.

As a final example, we should mention the work of Conklin et al ${ }^{(5)}$ on $\mathrm{PbTe}$ (Slide 5). This represents, in my opinion, the best perturbation-type calculation because the unperturbed wave functions were determined from a complete nonrelativistic energy band calculation. In this approach the authors first solved for the eigenfunctions and eigenvalues of the nonrelativistic hamiltonian using the APW (augmented plane wave) method $^{(6)}$. Linear combinations of these eigenfunctions were then used as trial functions in setting up the matrix elements of the relativistic hamiltonian. The roots of the resulting secular equation then yielded the relativistic energy levels.

The main disadvantage to the perturbation approach is that crystal wave functions are required and these are not easily acquired. An approach which avoids this was developed by $P$. Soven ${ }^{(7)}$ and first applied to the free-electron metal thallium (Slide 6). He generalized the OPW (orthogonalized plane wave) method by recasting it in the Dirac formalism. This involved orthogonalizing a 4-component Dirac plane wave to the 4component central field orbitals which represented the relativistic core states. This method includes in a very natural way all of the relativistic effects and does not require one to first determine the crystal wave functions if only the relativistic energy levels are desired. The method should be very useful for free-electrion metals, but suffers, as does the nonrelativistic OPW method, in that it is not practical for transition elements. 
It has been my experience in nonrelativistic band calculations $(9-11)$ that the APW method is much easier to use and more generally applicable than the OPW method. The crystal potential is straightforward to construct in the muffin-tin form, and the basis function includes in a very natural way all of the necessary orbital character even if it is not present in the core states. These, along with certain computational advantages, have led me to prefer the APW method. Thus when I became interested in relativistic effects in crystals, the first thing that suggested itself was to reformulate the APW method according to the Dirac theory. This led to a very convenient method for calculating relativistic energy bands which is applicable to both free-electron and transition elements.

We consider a typical unit cell in the crystal as being divided into two regions: a spherical region (I) inside of which the potential is spherically symmetric, and an outer region (II) in which the potential is assumed to be constant. In constructing a basis func-

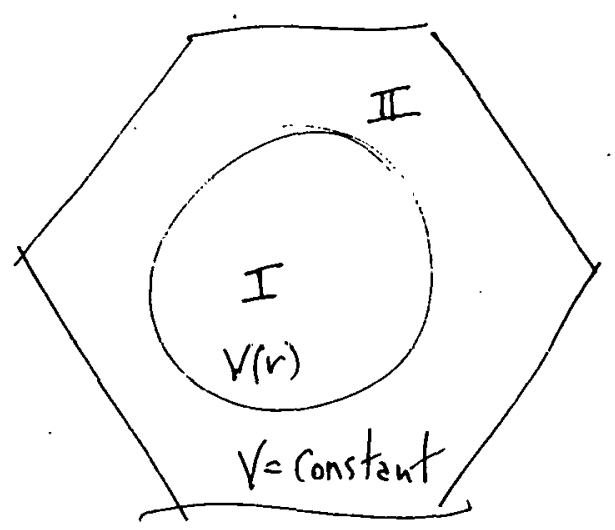
tion we shall form a linear combination of the central field Dirac orbitals in region I (Slides $7 \& 8$ ). In the outer region the basis function is taken to be a Dirac plane wave (written here in spherical coordinates) which automatically satisfies the Bloch condition imposed by the periodicity of the crystal potential (Slide 9). By an appropriate choice of the expansion 
coefficients occurring in the wave function in the central field region (I), it is possible to match the upper two components with the plane wave in region II over the spherical boundary. The two lower components, however, will in general be discontinuous over this surface. A variational expression for the energy which is valid for such a wave function has been constructed (Slide 10). The surface integral in this expression takes account of the discontinuity in the wave function across the spherical boundary. The wave function constructed in this fashion is called a RAPW (relativistic APW). As a trial function we consider a linear combination of these (a truncated reciprocal lattice expansion) and determine the coefficients from the variational principle. For details on this and the exact form of the resulting matrix elements you are referred to the original article on the subject ${ }^{(12)}$.

The RAPW method was originally developed in order to study relativistic effects in tungsten ${ }^{(13)}$. This metal has a body-centered cubic lattice; the Brillouin zone is shown on Slide 11. The two large pieces of the Fermi surface are centered at the symmetry points $\Gamma$ and $H$.

The piece at $\mathrm{H}$ is called the hole octahedron (Slide 12), and the piece at $\Gamma$ is called the electron jack (Slide 13). Cross-sections of these pieces of Fermi surface with the zone faces as determined by a nonrelativistic APW calculation are shown in Slide 14. We notice in this drawing that the "knob" on the electron jack contacts one of the corners of the hole octahedron about half way between $\Gamma$ and $H$. It has been found experimentally, however, by $\mathrm{Walsh}^{(4)}$ that there is in fact a gap between these 
two pieces of Fermi surface. The experimentally determined gap is about $5 \%$ of the distance $\Gamma \mathrm{H}$. The relativistic energy bands along this symmetry axis as determined by the RAPW method are shown on Slide 15. The distance $a b$ is an extremal dimension of the electron jack; the distance cd corresponds to the hole octahedron, and the gap bc between these two surfaces is found theoretically to be about $6 \frac{1}{2} \%$ of the distance $\Gamma \mathrm{H}$. This represents a very critical test of the theory and demonstrates the applicability of the RAPW method to heavy transition elements. One can go further and calculate the Fermi surface using this method (Slide 16). A careful comparison between this Fermi surface and other experimental results (in particular, de Haas-van Alphen measurements) is currently being carried out. Preliminary results indicate that the relativistic effects on the Fermi surface are quite pronounced and must be included in order to give agreement with the experimental results.

As an example of the effectiveness of the RAPW method to freeelectron metals, let us look at the results obtained for lead ${ }^{(14)}$. This is a particularly good test case because of the availability of very accurate and quite extensive experimental results. The most recent, and by far the most complete, study of the Fermi surface of lead has been reported by Anderson and Gold $(15,16)$. Based on extensive de Haas-van Alphen measurements, these authors have developed a four-parameter model of the Fermi surface which agrees remarkably well with the experimental results for this element. The energy bands predicted by this model are shown on Slide 1.7 along with the relativistic energy bands 
calculated using the RAPW method. The agreement at the Fermi energy is very good, and the spin-orbit splitting at the symmetry point $\mathrm{W}$ is manifested.

The agreement with experimental results in these two different applications of the RAPW method is quite favorable. It seems that the method should be applicable to all of the heavy elements (magnetic effects withstanding). Currently we are studying relativistic effects in lutecium and also in some of the semi-conductors with diamond structure. 


\section{References}

1. F. Herman, C. Kuglin, K. Cuff and R. Kortum, Phys. Rev. Letters 11, 541 (1963).

2. F. Herman, 1964. Lockheed Missiles and Space Company, Final Report, Contract NONR $3829(00)$.

3. L. F. Mattheiss and R. E. Watson, Phys. Rev. Letters 13, 526 (1964).

4. W. M. Walsh, Jr. and C. C. Grimes, Phys. Rev. Letters 13, 523 (1964).

5. J. B. Conklin, Jr., L. E. Johnson, and G. W. Pratt, Jr., Phys. Rev. 137, A1282 (1965).

6. J. C. Slater, Phys. Rev. 51, 846 (1937).

7. P. Soven, Phys. Rev: 137, A1706 (1965).

8. C. Herring and A. G. Hill, Phys. Rev. 58, 132 (1940).

9. T. L. Loucks and P. H. Cutler, Phys. Rev. 133, A819 (1964).

10. T. L. Loucks, Phyo. Rev. 134, Al618 (1964).

11. T. L. Loucks (APW Calculations for Cr, Mo and W to be published Phys. Rev.).

12. T. L. Loucks (to be published Phys. Rev.).

13. T. L. Loucks, Phys. Rev. Letters 14, 693 (1965).

14. T. L. Loucks (to be published Phys. Rev. Letters).

15. J. R. Anderson and A. V. Gold, 1963. United States Atomic Energy Commission, Research and Development Report No. IS-762.

16. J. R. Anderson and A. V. Gold (to be published Phys. Rev.). 


$$
\mathrm{H}=\bar{\alpha} \cdot \overline{\mathrm{p}}+\beta+\mathrm{V}
$$

$$
\begin{aligned}
\Im C=\frac{p^{2}}{2 m}+V+\frac{\hbar}{4 m^{2} c^{2}} \cdot[(\nabla V) \times \mathbf{p}] & \\
& +\frac{\hbar^{2}}{8 m^{2} c^{2}}\left(\nabla^{2} V\right)-\frac{1}{8 m^{3} c^{2}} p^{4}, \quad(1)
\end{aligned}
$$

3
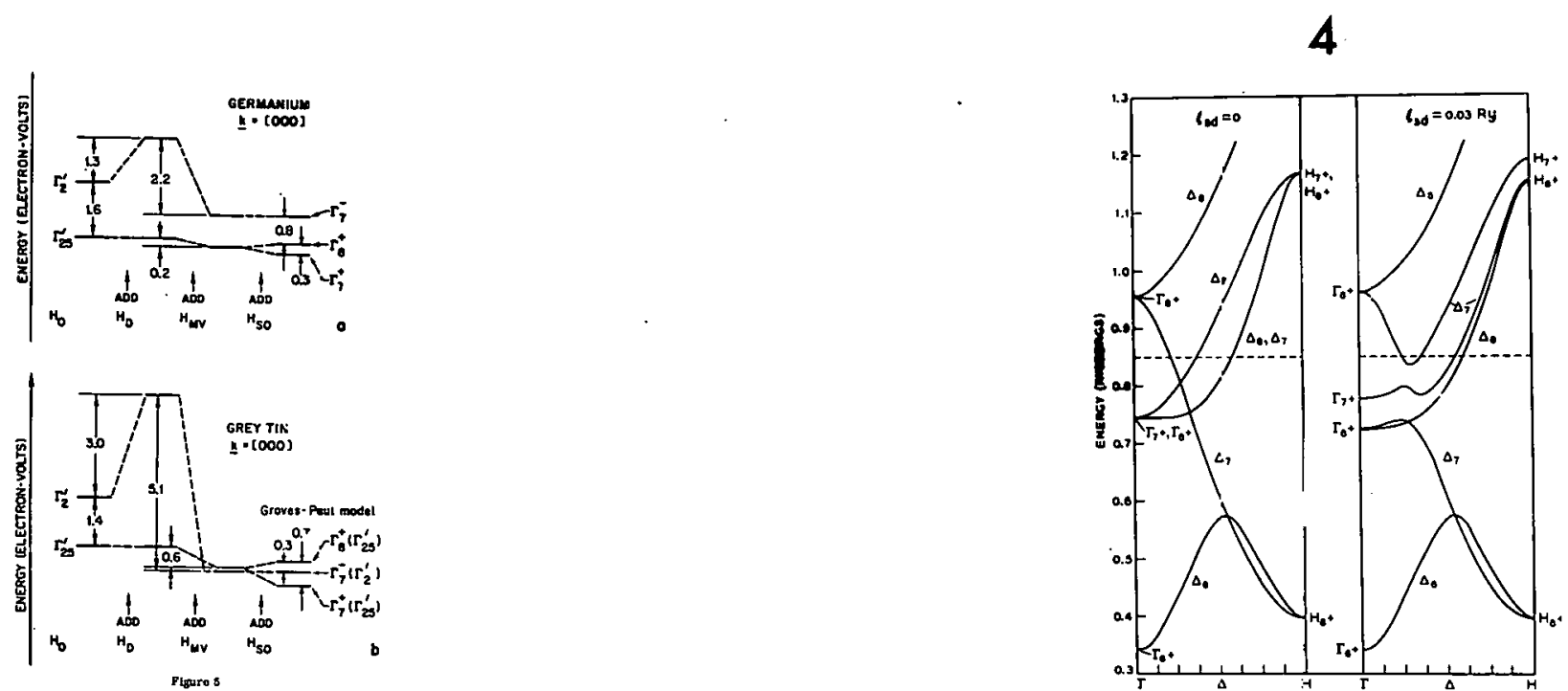

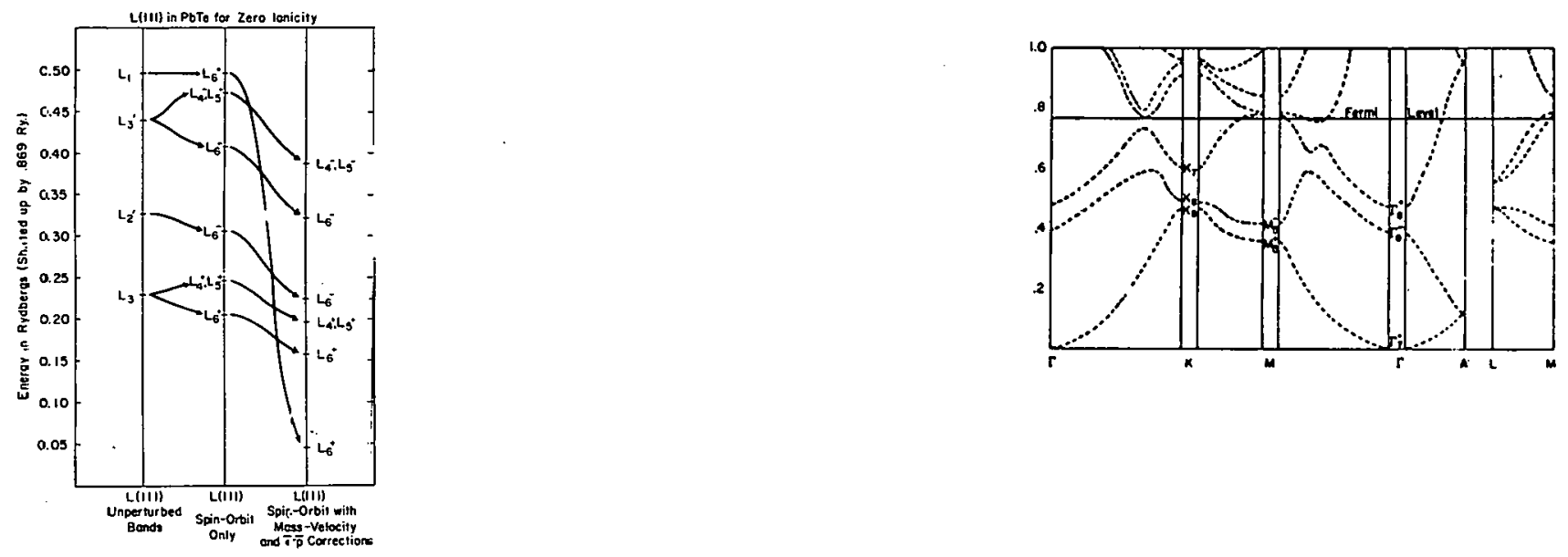

$$
\Psi_{n \mathrm{~m}}^{I}=\sum_{x \mu} A_{x \mu}^{\mathrm{nm}}\left(\begin{array}{cc} 
& \\
g_{x}(r) & x_{x}^{\mu} \\
i f_{x}(r) & x_{-x}^{\prime \prime \prime}
\end{array}\right)
$$

$$
\begin{aligned}
& \frac{d f}{d r}=\frac{(x-1) f}{r}-(W-1-V) g \\
& \frac{d g}{d r}=(W-V+1) f-\frac{(x+1)}{r} g
\end{aligned}
$$




$$
\underset{n m}{\Psi I I}=\sum_{x \mu} a_{x \mu}^{n m}\left(\begin{array}{ll}
j_{j}\left(k_{n} r\right) & x_{x}^{\mu} \\
\frac{i k_{n} S_{x}}{k_{n}^{*}+1} & j_{\ell}\left(k_{n} r\right) x_{-x}^{\mu}
\end{array}\right)
$$

11

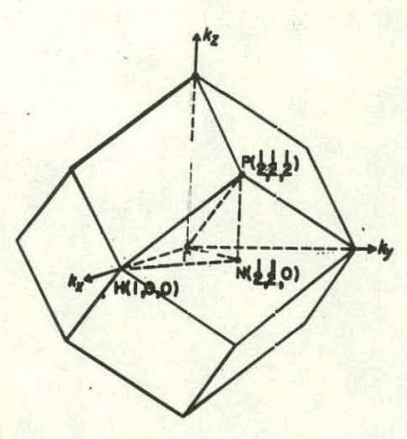

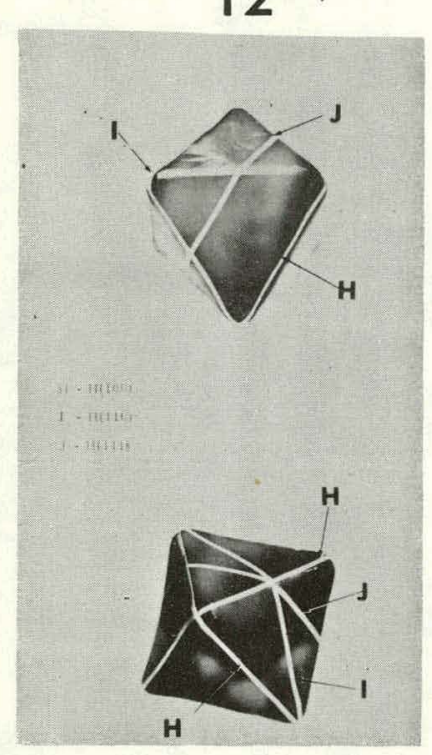




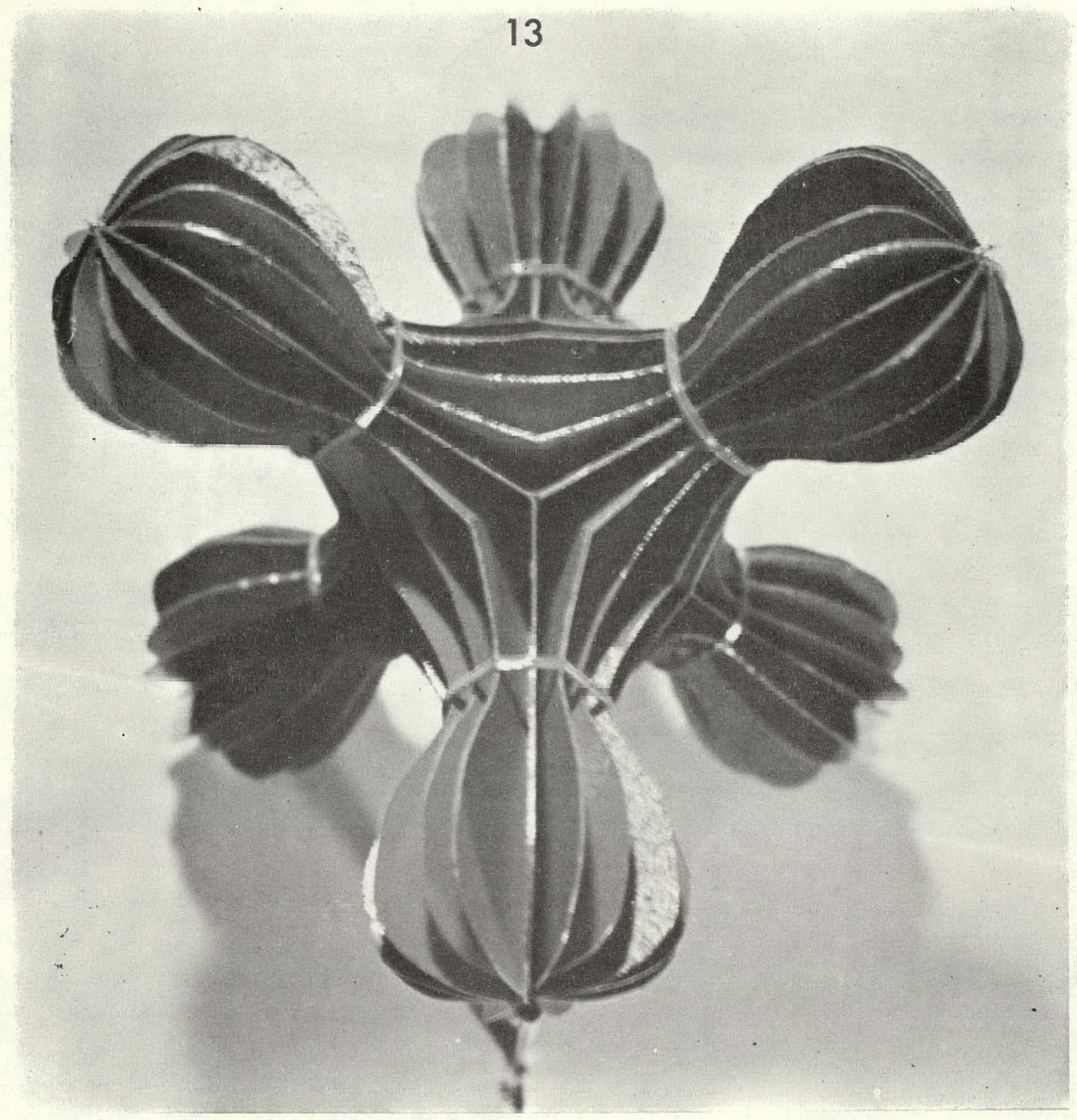



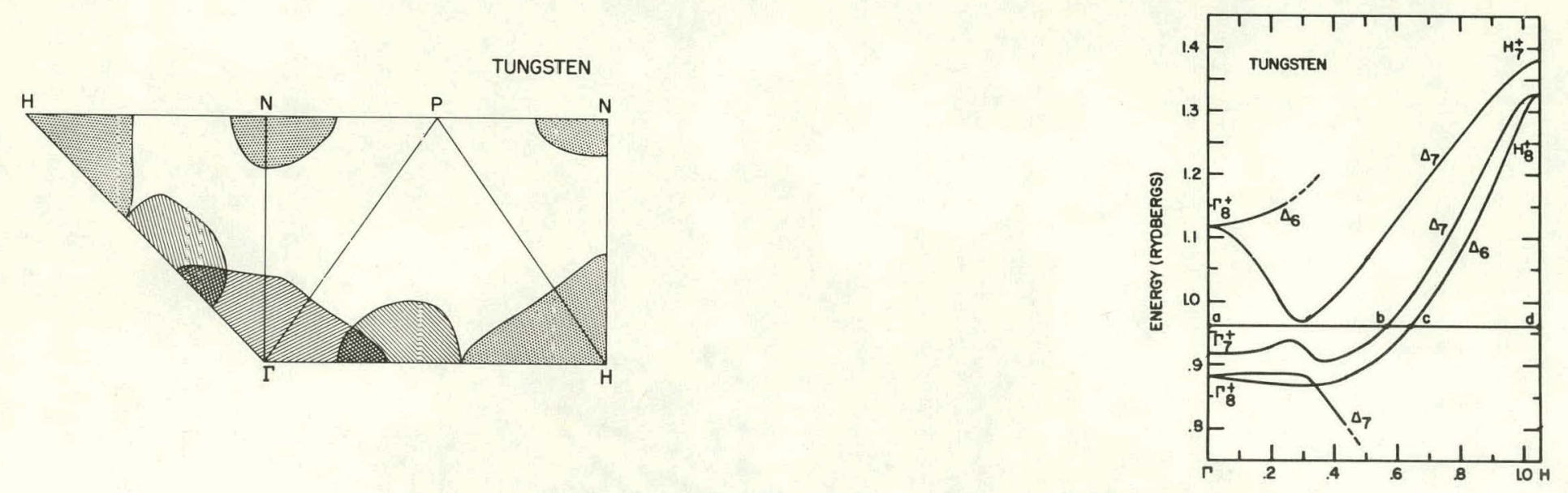

16

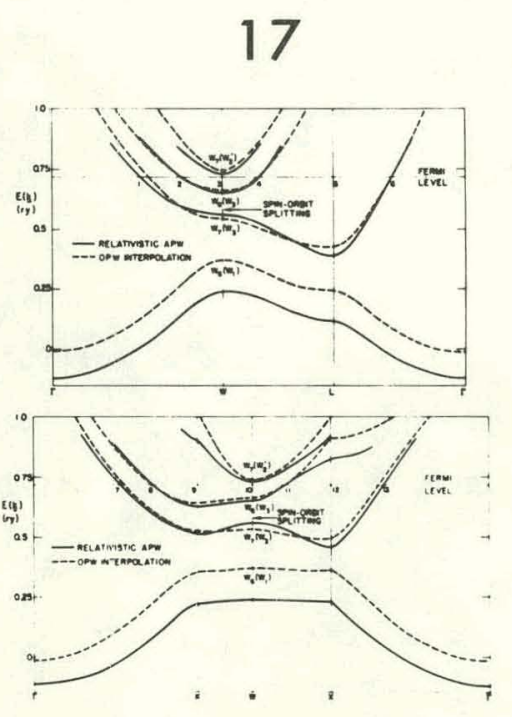

